\title{
Resistência de linhas de cola para madeiras de reflorestamento
}

\author{
Strength of glue line for reforestation wood
}

\author{
Pedro Gutemberg de Alcântara Segundinho'; Carlito Calil Neto²; Fabrício Gomes \\ Gonçalves ${ }^{3}$; Adair José Regazzi ${ }^{4}$; Carlito Calil Junior ${ }^{5}$
}

\section{Resumo}

Esse trabalho tem como objetivo avaliar a resistência ao cisalhamento na linha de cola de madeira laminada colada (MLC) fabricadas com três espécies florestais: Tectona grandis, Pinus oocarpa e Eucalyptus sp.. As colagens foram realizadas com lamelas sem qualquer tratamento químico preservativo, utilizando-se de adesivo bicomponente à base de melamina-ureia-formaldeído (MUF). Foram feitos ensaios de resistência ao cisalhamento na linha de cola para madeira nas condições de umidade de equilíbrio local e alta umidade, bem como ensaios de delaminação. Os resultados obtidos a partir dos ensaios de resistência ao cisalhamento na linha de cola na condição seca e dos ensaios de delaminação apontam para o melhor desempenho da espécie Pinus oocarpa. Concluiu-se que há potencialidade dessa espécie para produção de MLC utilizando o adesivo MUF.

Paravras chave: : Melamina-ureia-formaldeído. Tensão de cisalhamento. Delaminação.

\begin{abstract}
This paper aims to evaluate the shear strength of glued laminated timber (glulam) made of three wood species: Tectona grandis, Pinus oocarpa and Eucalyptus sp.. The bonds were made with lamellae with no chemical treatment, using two-component adhesive based on melamine-urea-formaldehyde (MUF). Glue line shear tests were made for wood in local moisture content and high humidity, as well as delamination tests. The results presented in the glue line shear strength tests in dry condition and delamination for the three species indicate better performance of Pinus oocarpa. It was concluded that this species there is potential to produce MLC utilizing the MUF adhesive.

Keywords: melamine-urea-formaldehyde, shear strength, delamination.
\end{abstract}

Keywords: Melamine-urea-formaldehyde. Shear strength. Delamination.

\footnotetext{
${ }^{1}$ Prof. Dr., Departamento de Ciências Florestais e da Madeira, Centro Ciências Agrárias, Universidade Federal do Espírito Santo, Av. Gov. Lindemberg, 316, 29550-000, Jerônimo Monteiro, ES, Brasil. E-mail: p_gutemberg2001@yahoo.com.br

${ }^{2}$ Doutor, Departamento de Engenharia de Estruturas, Escola de Engenharia de São Carlos, Universidade de São Paulo, Av. Trabalhador São-carlense, 400, 13566-590, São Carlos, SP, Brasil. E-mail: netousp@gmail.com

${ }^{3}$ Prof. Dr., Departamento de Ciências Florestais e da Madeira, Centro Ciências Agrárias, Universidade Federal do Espírito Santo, Av. Gov. Lindemberg, 316, 29550-000, Jerônimo Monteiro, ES, Brasil. E-mail: fabricio.goncalves@ufes.br

${ }^{4}$ Prof. Titular (Aposentado), Departamento de Estatística, Universidade Federal de Viçosa, Campus Universitário, 36570-000, Viçosa, MG, Brasil. E-mail: adairreg@ufv.br

${ }^{5}$ Professor Titular, Departamento de Engenharia de Estruturas, Escola de Engenharia de São Carlos, Universidade de São Paulo, Av. Trabalhador São-carlense, 400, 13566-590, São Carlos, SP, Brasil. E-mail: calil@sc.usp.br
} 


\section{Introdução}

A Madeira Laminada Colada (MLC) é um produto engenheirado de madeira utilizado em construções na forma de elementos estruturais, que requerem precisão de fabricação em todos os seus estágios de tal maneira a garantir segurança quando solicitados pelas ações permanentes e variáveis. $\mathrm{Na}$ literatura relacionada à MLC, encontra-se que é possível utilizar todas as espécies florestais madeireiras na colagem de MLC, segundo Moody, Hernandez, e Liu (1999). Entretanto, algumas espécies madeireiras possuem características físicas e químicas que exigem o emprego de colas especiais ou a modificação das colas já comercializadas por meio de misturas (SANTOS; DEL MENEZZI, 2010).

Os produtos de MLC acabados podem ser testados somente em condições laboratoriais. Logo, é necessário controlar a qualidade da produção, tal como recomenda a norma canadense (CANADIAN STANDARDS ASSOCIATION - CAN/CSA 0177, 2006), para assegurar que as propriedades sejam adequadas para o emprego na construção civil. Quanto ao sistema de controle de qualidade a ser implementado em uma indústria, este é definido pelas ações realizadas por um fabricante em relação aos materiais, métodos, equipamentos, mão de obra e produto final, para satisfazer os requisitos necessários de uma norma de controle de qualidade, a exemplo do recomendado e especificado pelo American Institute of Timber Construction (AITC $\mathrm{T} 115,2004)$.

No Brasil, como o produto MLC ainda é pouco utilizado nas construções, é necessário que os estudos devam ser realizados no sentido de se proceder, em cada Região ou Estado, uma investigação de interdependência entre espécie-adesivo-umidade para uma caracterização das madeiras que melhor possam se adaptar a essa técnica, a fim de serem produzidos elementos estruturais de MLC que possam ser utilizados em ambientes exterior ou interior conforme sugerido pela The Engineered
Wood Association (APA, 2002). As espécies mais utilizadas para o emprego em MLC são pertencentes ao grupo das coníferas porque são abundantes nos países do hemisfério norte, berço da MLC (SZÜCS, 2006). No entanto há algumas espécies folhosas nativas do Brasil cuja potencialidade já foi testada, conforme Dias e Lahr (1999), Macedo, Dias e Barata (2000) e Zangiácomo (2003).

Um programa de qualidade em MLC conta com vários especialistas que entendem da importância do processo de certificação para conseguir a confiança do consumidor e a qualidade final do produto. Para predizer o desempenho estrutural da MLC com base no programa de qualidade, é necessária a realização de ensaios mecânicos diários, sistemas de avaliação em pontos estratégicos da produção e inspeção constante durante o processo de produção. Segundo Faherty e Williamson (1997), a utilização de ensaios de cisalhamento na linha de cola é uma forma eficiente para testar a resistência das ligações realizadas por adesivos. Para a fabricação de elementos estruturais de MLC, devem ser usados apenas adesivos estruturais resistentes às intempéries. Destaca-se que todas as atividades envolvendo a fabricação de MLC devem ser auditadas e verificadas por inspetores credenciados.

Quanto aos adesivos, no início do século XX, utilizava-se a caseína, adesivo natural derivado do leite; em meados do mesmo século, surgiram os adesivos sintéticos, e mais recentemente a melamina-ureia-formaldeído, também sintético. No entanto, a utilização dos adesivos depende mais das condições de uso dos elementos estruturais que do tipo da madeira. Logo, é preciso levar em consideração principalmente o meio no qual a estrutura vai estar exposta, principalmente, temperatura e teor de umidade. De acordo com Tienne et al. (2011), apesar das diversas formulações de adesivos desenvolvidas, no Brasil, são escassos os trabalhos com o objetivo de testar a resistência do material e as condições de intemperismo. Não há dúvida de que a utilização da madeira deve ter destaque nessas investigações, principalmente as 
madeiras provenientes de florestas plantadas, tais como as espécies Eucalyptus e Pinus, e atualmente a espécie Teca.

O Anuário Estatístico da Associação Brasileira de Produtores de Florestas Plantadas (ABRAF, 2012) destaca que a Teca (Tectona grandis) é nativa das florestas tropicais índicas e asiáticas. No Brasil, é plantada em escala comercial nos estados do Mato Grosso, Pará e Roraima. A madeira da espécie é utilizada principalmente na construção civil (portas, janelas, lambris, painéis, forros), assoalhos e deques, móveis, embarcações e lâminas decorativas. A área de plantios florestais com Teca no Brasil cresceu nos últimos três anos 58.711 ha (2009), 65.440 ha (2010) e 67.693 ha (2011). Quanto à distribuição da área total plantada de Eucalyptus e Pinus das associadas individuais da ABRAF por segmento industrial, destaca-se a participação dos segmentos de Papel e Celulose, Siderurgia a Carvão Vegetal, Painéis de Madeira Industrializada e Produtores Independentes com $71,2 \%, 18,4 \%, 6,8 \%$ e 3,6\%, respectivamente, para o Eucalyptus; com $61,1 \%$, $5,1 \%, 20,6 \%$ e $13,3 \%$, respectivamente, para o Pinus. Em 2011, a área ocupada por plantios florestais de Eucalyptus e Pinus no Brasil totalizou 6.515.844 ha, sendo 74,8\% correspondente à área de plantios de Eucalyptus e $25,2 \%$ aos plantios de Pinus. Nesse ano, não houve crescimento da área de plantios florestais de Eucalyptus e Pinus no Brasil, pois o aumento de área apurado (5.151 ha, 0,1\%) está dentro da margem de erro do levantamento. O indicador de 2011 corrobora a tendência de desaceleração do crescimento da área de plantios apresentada nos dois anos anteriores. No período 2005-2011, o crescimento acumulado foi de 27,9\%, ou seja, 3,0\% ao ano.

O trabalho tem como objetivo avaliar a resistência ao cisalhamento na linha de cola de madeira laminada colada (MLC) fabricadas com três espécies de madeira de reflorestamento não tratadas contra organismos xilófagos: Tectona grandis, Pinus oocarpa e Eucalyptus sp.. O artigo tem por finalidade um estudo inicial da combinação de espécie-adesivo-umidade dessas espécies, utilizando-se adesivo à base de melamina-ureiaformaldeído para a colagem das lamelas, visando posteriormente o desenvolvimento de elementos estruturais a serem aplicados na construção civil, uma vez que, ainda são pouco utilizados no Brasil.

\section{Material e métodos}

Fabricação das vigas de $M L C$

Para a produção das vigas de MLC, foram utilizadas madeiras das espécies Tectona grandis, com idades de 4, 8 e 12 anos da região de Catalão, estado de Goiás; Pinus oocarpa com idades de 8 e 12 anos da região de Catalão, estado de Goiás; e Eucalyptus sp. com idade de 14 anos da região de Brotas, estado de São Paulo. Todas essas madeiras foram extraídas a partir de florestas renováveis de árvores plantadas no Brasil. Para cada espécie foram produzidas vigas de MLC, com lamelas de madeira, sem tratamento preservativo, utilizando-se o adesivo bicomponente de aplicação estrutural à base de melamina-ureia-formaldeído (Akzo Nobel MUF 1242/2542) com catalisador na proporção de 100 partes/peso do adesivo e 20 partes/peso do endurecedor.

$\mathrm{O}$ adesivo foi aplicado em ambas as faces das lamelas com gramaturas de 200-250 g.m-2 (linha dupla de cola) e pressão de colagem mínima de 1,0 $\mathrm{MPa}$ tomada para madeiras de densidade elevada, conforme recomendações do fabricante do adesivo. Foram produzidas três vigas de MLC de cada espécie, sendo cada viga com três lamelas de aproximadamente $30 \mathrm{~mm}$ de espessura, distribuídas aleatoriamente com aplicação de pressão de 1,0 MPa em uma prensa tipo "A". Todas as vigas de MLC tinham comprimento de $200 \mathrm{~cm}$ e seção transversal de $9 \mathrm{~cm}$ x $9 \mathrm{~cm}$ para a madeira de Tectona grandis e Pinus oocarpa, e $9 \mathrm{~cm}$ x 5,5 $\mathrm{cm}$ para a madeira de Eucalyptus sp.. A largura das vigas de MLC foi tomada conforme a disponibilidade das dimensões 
das peças de madeira. A Figura 1 ilustra a forma de colagem das lamelas de madeira na confecção das vigas estruturais de MLC. Nessa técnica de colagem por meio de prensa hidráulica é possível fazer a colagem de até 10 vigas de MLC com $9 \mathrm{~cm}$ de altura cada, mantendo-se a pressão com 1,0 MPa durante o tempo de cura do adesivo por meio de ajuste automático de fluxo constante de óleo nos cilindros hidráulicos. O tempo de cura do adesivo variou entre 10 horas a $20^{\circ} \mathrm{C}$ e 3 horas a $30^{\circ} \mathrm{C}$, máximo e mínimo respectivamente.

Figura 1. Montagem das vigas de madeira laminada colada (MLC)

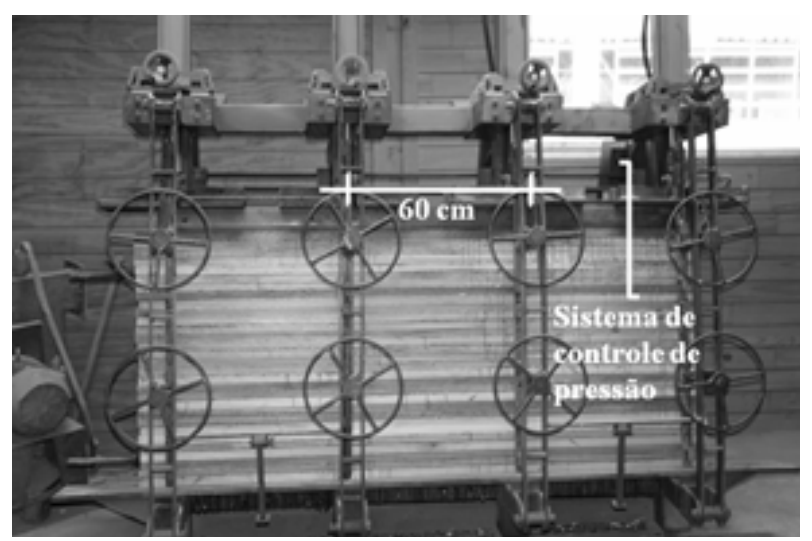

Fonte: Autores

\section{Ensaios de cisalhamento e delaminação}

Para esse estudo foram feitos os ensaios de cisalhamento na linha de cola (fv0) na máquina universal Amsler, em corpos de prova na condição seca, segundo a norma brasileira NBR 7190 (ABNT, 1997). $\mathrm{O}$ ensaio de cisalhamento (fv0) também foi realizado em corpos de prova estruturais cujas dimensões são da seção transversal das vigas de MLC (Figura 2a), conforme apresentado por Frangi, Fontana e Mischler (2004), nas condições seca e úmida, respectivamente seco ao ar e umedecida após 48 horas imersos em água à temperatura ambiente. Foram realizados ensaios de cisalhamento em madeira maciça na condição seca, segundo a norma brasileira NBR 7190 (ABNT, 1997), para efeito de comparação com a madeira em MLC. A qualidade da viga estrutural confeccionada em MLC foi ainda avaliada com base no ensaio de delaminação conforme apresentado na Figura $2 b$, segundo a recomendação AITC T110 (2007). Esse ensaio avaliou o comportamento da combinação espécie-adesivo para uso exterior mediante autoclavagem, durante ensaio que requer 12 dias para completar os 3 ciclos. O primeiro ciclo totaliza um período de 96 horas, (4 dias). Nos segundo e terceiro ciclos são repetidas as mesmas etapas do primeiro ciclo. Cada ciclo é composto de duas etapas. Inicialmente, na etapa um do primeiro ciclo, aplica-se um vácuo de $75 \pm 10 \mathrm{kPa}$ por um período não inferior a 2 horas, depois o vácuo deve ser liberado e aplica-se água com pressão de $540 \pm 20 \mathrm{kPa}$, também por um período não inferior a 2 horas, certificando-se que os corpos de prova estão submersos. Na etapa dois do primeiro ciclo, repetem-se os mesmos procedimentos da etapa um, totalizando 8 horas de permanência na autoclave. Após o período de ensaios na autoclave, as amostras de 76,2 mm de comprimento são secas ao ar livre durante 88 horas à temperatura de $28 \pm 2^{\circ} \mathrm{C}$ visando à redução de seu peso na faixa de 5 a $6 \%$ do peso da amostra inicial. Recomenda-se que a delaminação, medida por meio de paquímetro, total das linhas de cola de cada corpo de prova não deve exceder $1 \%$ do comprimento total de linhas de cola no topo de todos os corpos de prova para que esta MLC esteja aprovada para o uso exterior. As recomendações para uso interior e exterior foram tomadas a partir da The Engineered Wood Association (APA, 2002).

Figura 2. Corpo de prova estrutural para ensaio de cisalhamento (a) e corpo de prova de delaminação (b)

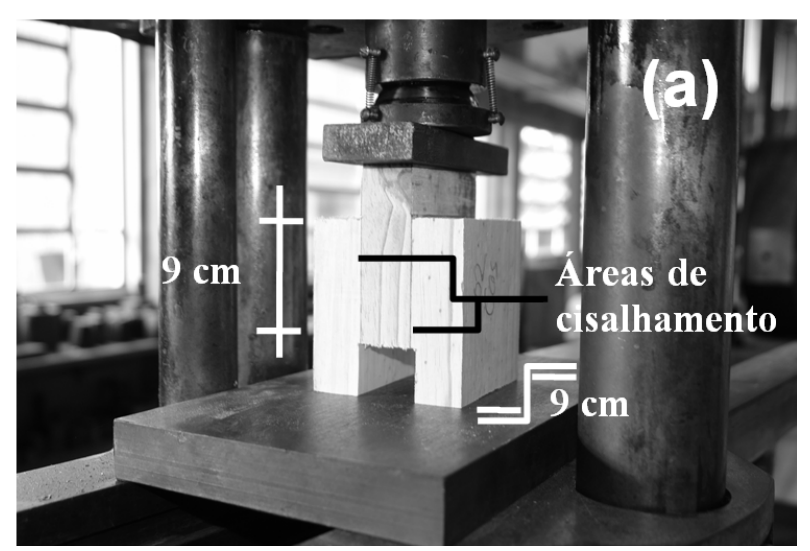




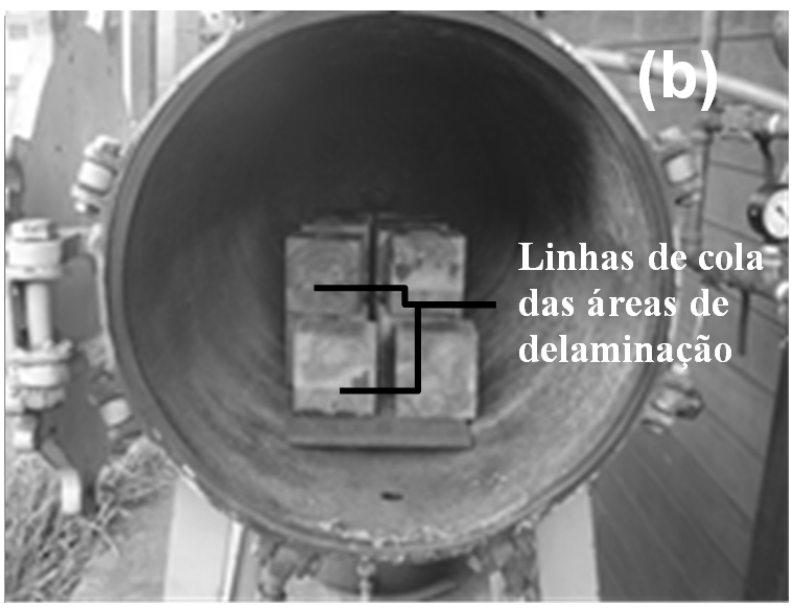

Fonte: Autores

\section{Análise estatística dos dados}

Os tratamentos avaliados para as três espécies com o respectivo número de corpos de prova de cisalhamento estão apresentados na Tabela 1 . Retirou-se das lamelas no mínimo um corpo de prova de cisalhamento antes da produção das vigas de MLC, dessa forma tem-se maior número de repetições, Tabela 1 .

Tabela 1. Número de corpos de prova para obtenção da resistência média ao cisalhamento

\begin{tabular}{cccc}
\hline Ensaios de resistência & \multicolumn{3}{c}{ Espécies } \\
\cline { 2 - 4 } ao cisalhamento & Tectona grandis & Pinus oocarpa & Eucalyptus sp. \\
\hline$[\mathrm{I}] \mathbf{f}_{\mathrm{v} 0}$ (madeira sólida) & 142 & 184 & 72 \\
{$[\mathrm{II}] \mathbf{f}_{\mathrm{v} 0}$ (MLC seca) } & 6 & 6 & 9 \\
{$[\mathrm{III}] \mathbf{f}_{\mathrm{v} \mathbf{0}}$ (MLC seca) } & 3 & 3 & 3 \\
{$[\mathrm{IV}] \mathbf{f}_{\mathrm{v} 0}$ (MLC úmida) } & 3 & 3 & 3 \\
\hline
\end{tabular}

[I] e [II] corpos de prova segundo a NBR 7190 (ABNT, 1997); [III] e [IV] corpos de prova estrutural conforme Frangi, Fontana e Mischler (2004).

Os dados de cada espécie apresentada na Tabela 1 foram submetidos ao teste de normalidade (SHAPIRO; WILK, 1965) e ao teste de homogeneidade de variâncias (LEVENE, 1960). Para as espécies Pinus oocarpa e Eucalyptus $s p$., em que as pressuposições foram satisfeitas, realizou-se uma análise de variância (ANOVA) considerando o delineamento inteiramente casualizado com quatro tratamentos. As médias foram comparadas pelo teste de Tukey em nível de $5 \%$ de probabilidade. Para os dados de Tectona grandis, a hipótese de normalidade foi rejeitada, portanto não atendendo às pressuposições da análise de variância (ANOVA). Logo, optou-se pela utilização de um teste não paramétrico que independe da distribuição dos dados. Neste caso, na comparação dos quatro tratamentos foi aplicado o teste de Kruskal-Wallis; e na comparação de dois tratamentos, o teste de Mann-Whitney. Salientase que não foram feitas comparações entre as três espécies e sim dentro de cada espécie, uma vez que são espécies diferentes, com densidades diferentes e com características anatômicas e químicas diferentes em sua composição percentual dividida entre componentes estruturais e não estruturais na formação da madeira. Nesse trabalho foram obtidos os valores das densidades aparentes iguais a 556 kg.m-3, 608 kg.m-3 e 872 kg.m-3 para as espécies Tectona grandis, Pinus oocarpa e Eucalyptus sp., respectivamente, conforme a NBR 7190 (ABNT, 1997).

Para os ensaios de delaminação foram tomados seis corpos de prova de cada espécie. Os dados foram avaliados considerando apenas estatísticas descritivas.

\section{Resultados e discussão}

Na Tabela 2 são apresentados os resultados dos testes de Kruskal-Wallis e Mann-Whitney, para os dados de cisalhamento da espécie Tectona grandis. Optou-se pelo teste não paramétrico, pois os dados não atendem as pressuposições para realização de uma ANOVA e teste paramétrico. 
Tabela 2. Resultados do teste (Kruskal-Wallis) e (Mann-Whitney), para os dados de cisalhamento da espécie Tectona grandis

\begin{tabular}{|c|c|c|}
\hline \multicolumn{3}{|c|}{ Tectona grandis } \\
\hline Tratamentos & Média (MPa) & Teste de Kruskal-Wallis \\
\hline [I] $\mathbf{f}_{\mathrm{v} 0}$ (madeira sólida) & 10,01 & $\chi^{2}=20,2917^{* *}$ \\
\hline [II] $\mathbf{f}_{\mathrm{v} \theta}$ (MLC seca) & 8,61 & G.L. $=3$ \\
\hline [III] $\mathbf{f}_{\mathrm{v} 0}$ (MLC seca) & 6,09 & Valor $-\mathrm{P}=0,0001$ \\
\hline [IV] $\mathbf{f}_{\mathbf{v} 0}$ (MLC úmida) & 4,65 & \\
\hline Comparaçôes & Estatistica & Teste de Mann-Whitney (Valor-P) \\
\hline [I] vs. [II] & $217,5^{*}$ & 0,0260 \\
\hline [I] vs. [III] & $16,0^{* *}$ & 0,0049 \\
\hline [I] vs. [IV] & $15,0^{* *}$ & 0,0047 \\
\hline [II] vs. [III] & $7,0^{\text {n.s. }}$ & 0,0528 \\
\hline [II] vs. [IV] & $6,0^{*}$ & 0,0282 \\
\hline [III] vs. $[\mathrm{IV}]$ & 15,0 , n.5. & 0,0809 \\
\hline
\end{tabular}

**Significativo em nível de $1 \%$ de probabilidade $(\mathrm{P}<$ $0,01)$; *Significativo em nível de $5 \%$ de probabilidade (P $<0,05)$; n.s.Não significativo (P > 0,05); [I] e [II] corpos de prova segundo a NBR 7190 (ABNT, 1997); [III] e [IV] corpos de prova estrutural, Frangi, Fontana e Mischler (2004).

Com os resultados apresentados na Tabela 2, constatou-se que na espécie Tectona grandis, as médias obtidas nos tratamentos [I] e [II] diferiram significativamente $(\mathrm{P}<0,05)$, enquanto que os tratamentos [III] e [IV] não diferiram significativamente $(\mathrm{P}>0,05)$. Nessa espécie, o corpo de prova estrutural de MLC na condição seca, tratamento [III], foi capaz de representar a resistência ao cisalhamento estimado no tratamento [II], pois não diferiram significativamente $(\mathrm{P}>0,05)$. Logo, a resistência da junta colada diferiu estatisticamente da resistência ao cisalhamento longitudinal da madeira nos tratamentos [I] e [II], quando deveria ser no mínimo igual, segundo a NBR 7190 (ABNT, 1997).

Nas Tabelas 3 e 4 são apresentados os resultados da análise de variância, teste de normalidade, teste de homogeneidade de variâncias e teste de médias para os dados de cisalhamento das espécies Pinus oocarpa e Eucalyptus sp., respectivamente. Tanto a hipótese de normalidade quanto a hipótese de homogeneidade de variâncias não foram rejeitadas, optou-se pelo teste paramétrico. Foi utilizada a média harmônica do número de repetições para obtenção da diferença mínima significativa para comparações das médias.

Tabela 3. Resultados da análise de variância, teste de normalidade (SHAPIRO; WILK, 1965), teste de homogeneidade de variâncias (Levene, 1960) e teste de médias (Tukey) para os dados de cisalhamento da espécie Pinus oocarpa

\begin{tabular}{|c|c|c|c|c|}
\hline \multirow{2}{*}{ F.V. } & \multicolumn{4}{|c|}{ Pinus oocarpa } \\
\hline & G.L. & Q.M. & $\mathrm{F}$ & Valor-P \\
\hline Tratamentos & 3 & 32,83 & $7,97^{* *}$ & $<0,0001$ \\
\hline Resíduo & 192 & 4,12 & & \\
\hline \multicolumn{5}{|l|}{ C.V. $=25,76 \%$} \\
\hline \multicolumn{5}{|l|}{ Média $=7,88 \mathrm{MPa}$} \\
\hline Shapiro-Wilk & $\mathrm{W}=0,992162^{\mathrm{n} . \mathrm{s}}$ & & Valor-P & \\
\hline Teste de Levene & $\mathrm{F}=1,22^{\mathrm{ns} .}$ & & Valor- $\mathrm{P}=$ & \\
\hline Tratamentos & \multicolumn{4}{|c|}{ Médias $(\mathrm{MPa})^{[1]}$} \\
\hline [I] $\mathbf{f}_{\mathbf{v} 0}$ (madeira sólida) & 8,01 & & A & \\
\hline$[I I] \mathbf{f}_{v 0}(\mathrm{MLC}$ seca) & 7,57 & & A & \\
\hline$[I I I] \mathbf{f}_{\mathrm{v} 0}$ (MLC seca) & 5,24 & & $\mathrm{AB}$ & \\
\hline [IV] $\mathrm{f}_{\mathrm{v} 0}$ (MLC úmida) & 2,92 & & B & \\
\hline
\end{tabular}

**Significativo ao nível de $1 \%$ de probabilidade (P $<0,01)$; n.s.Não significativo $(\mathrm{P}>0,05)$; rh é a média harmônica do número de repetições; D.M.S. é a diferença mínima significativa; [1]Em cada coluna, as médias seguidas de pelo menos uma mesma letra não diferem estatisticamente em nível de $5 \%$ de probabilidade pelo teste de Tukey; [I] e [II] corpos de prova segundo a NBR 7190 (ABNT, 1997); [III] e [IV] corpos de prova estrutural, Frangi, Fontana e Mischler (2004).

Com os resultados apresentados na Tabela 3, constatou-se que na espécie Pinus oocarpa, as médias obtidas nos tratamentos [I], [II] e [III] não diferiram significativamente entre si $(\mathrm{P}>0,05)$, enquanto que o tratamento [IV] diferiu significativamente ( $\mathrm{P}$ $<0,05)$ dos tratamentos [I] e [II]. Nessa espécie, o corpo de prova estrutural de MLC na condição seca, tratamento [III], foi capaz de representar as resistências ao cisalhamento estimadas nos tratamentos [I] e [II]. Logo, a resistência da junta colada não diferiu estatisticamente da resistência ao cisalhamento longitudinal da madeira nos tratamentos [I], [II] e[III], portanto em conformidade com a recomendação da NBR 7190 (ABNT, 1997). 
Tabela 4. Resultados da análise de variância, teste de normalidade (SHAPIRO; WILK, 1965), teste de homogeneidade de variâncias (Levene, 1960) e teste de médias (Tukey) para os dados de cisalhamento da espécie Eucalyptus $s p$.

\begin{tabular}{|c|c|c|c|c|}
\hline \multirow{2}{*}{ F.V. } & \multicolumn{4}{|c|}{ Eucalyptus sp. } \\
\hline & G.L. & Q.M. & $\mathrm{F}$ & Valor-P \\
\hline Tratamentos & 3 & 79,65 & $18,74 * *$ & $<0,0001$ \\
\hline Resíduo & 83 & 4,25 & & \\
\hline \multicolumn{5}{|l|}{ C.V. $=19,12 \%$} \\
\hline \multicolumn{5}{|l|}{ Média $=10,78 \mathrm{MPa}$} \\
\hline Shapiro-Wilk & $W=0,991948^{n . s}$ & & Valor- $\mathrm{P}=0$ & \\
\hline Teste de Levene & $\mathrm{F}=0,83^{\text {n.s. }}$ & & Valor- $\mathrm{P}=0$ & \\
\hline Tratamentos & \multicolumn{4}{|c|}{ Médias $(\mathrm{MPa})^{[1]}$} \\
\hline [I] $\mathrm{f}_{\mathrm{v} 0}$ (madeira sólida) & 11,49 & & A & \\
\hline [II] $\mathbf{f}_{\mathbf{v} 0}$ (MLC seca) & 8,47 & & $\mathrm{AB}$ & \\
\hline [III] $\mathrm{f}_{\mathrm{v} 0}$ (MLC seca) & 6,44 & & $\mathrm{BC}$ & \\
\hline$[\mathrm{IV}] \mathbf{f}_{\mathrm{r} 0}$ (MLC ümida) & 5,07 & & $\mathrm{C}$ & \\
\hline
\end{tabular}

**Significativo em nível de $1 \%$ de probabilidade (P $<0,01$ ); n.s.Não significativo ( $\mathrm{P}>0,05)$; rh é a média harmônica do número de repetições; D.M.S. é a diferença mínima significativa; [1]Em cada coluna, as médias seguidas de pelo menos uma mesma letra não diferem estatisticamente ao nível de $5 \%$ de probabilidade pelo teste de Tukey; [I] e [II] corpos de prova segundo a NBR 7190 (ABNT, 1997); [III] e [IV] corpos de prova estrutural, Frangi, Fontana e Mischler (2004).

Com os resultados apresentados na Tabela 4, constatou-se que na espécie Eucalyptus sp., as médias obtidas nos tratamentos [I] e [II] não diferiram significativamente $(\mathrm{P}>0,05)$, enquanto que os tratamentos [III] e [IV] diferiram significativamente $(\mathrm{P}<0,05)$ do tratamento [I]. Nessa espécie, o corpo de prova estrutural de MLC na condição seca, tratamento [III], não foi capaz de representar as resistências ao cisalhamento estimadas nos tratamento [I] e [II]. Logo, a resistência da junta colada não diferiu estatisticamente da resistência ao cisalhamento longitudinal da madeira nos tratamentos [I] e [II], portanto em conformidade com a recomendação da NBR 7190 (ABNT, 1997).

Os resultados das resistências ao cisalhamento nas três espécies obtidos por meios dos corpos de prova estrutural (FRANGI; FONTANA; MISCHLER, 2004), tratamentos [III] e [IV], são representativos, conforme apresentados nas Tabelas 2 a 4.
$\mathrm{Na}$ Tabela 5 são apresentadas as resistências relativas das tensões de cisalhamento para as três espécies de madeira. A resistência relativa foi calculada dividindo-se o valor médio das resistências das amostras de MLC pelo valor médio das resistências das amostras da madeira sólida.

Tabela 5. Resistências relativas de cisalhamento

\begin{tabular}{cccc}
\hline \multirow{3}{*}{ Ensaios } & \multicolumn{3}{c}{ Resistência Relativa } \\
\cline { 2 - 4 } & Tectona grandis Pinus oocarpa Eucalyptus sp. \\
\hline$[\mathrm{II}][\mathrm{I}]$ & 0,86 & 0,95 & 0,74 \\
\hline$[\mathrm{II}] /[\mathrm{I}]$ & 0,61 & 0,65 & 0,56 \\
\hline$[\mathrm{IV}] /[\mathrm{I}]$ & 0,46 & 0,36 & 0,44 \\
\hline
\end{tabular}

[I] corpo de prova de madeira sólida segundo a NBR 7190 (ABNT, 1997); [II] corpos de prova de MLC segundo a NBR 7190 (ABNT, 1997); [III] e [IV] corpos de prova estrutural de MLC, seco e úmido, respectivamente, conforme Frangi, Fontana e Mischler (2004).

A partir dos resultados das resistências relativas de cisalhamento apresentados na Tabela 5, todos em razão da madeira sólida, foi possível perceber que a espécie Pinus oocarpa teve o melhor desempenho na condição seca, relação [II]/[I], e o pior desempenho na condição úmida, relação [IV]/[I]. Observou-se que ocorreu, predominantemente, ruptura na madeira para o corpo de prova estrutural na condição seca e úmida para as três espécies; entretanto, o modo de ruptura não ocorreu no plano de cisalhamento, portanto necessita ser avaliado e estudado levandose em conta as relações geométricas do corpo de prova estrutural apresentado por Frangi, Fontana e Mischler (2004). As evidências mostraram que a ruptura foi provocada pela abertura inferior da lâmina central do corpo de prova estrutural, ou seja, não ocorreu cisalhamento puro e simples, havendo outro tipo de tensão devido à presença de um momento fletor ou outro tipo de solicitação que leve à abertura na parte inferior do corpo de prova $\mathrm{e}$ consequentemente a tensão de ruptura do corpo de prova diminuiu conforme foi observado nos ensaios. O corpo de prova estrutural sugerido por Frangi, 
Fontana e Mischler (2004) foi provavelmente ensaiado de forma inadequada, pois a resistência ao cisalhamento encontra-se na faixa de 34,58$43,95 \%$ inferior ao corpo de prova de cisalhamento da NBR 7190 (ABNT, 1997) na condição seca, e nesse caso deve-se desconsiderar os valores encontrados e recomendar que sejam feitas novas pesquisas levando em consideração principalmente as relações geométricas e as condições de fixação da parte inferior do corpo de prova no sistema de ensaio.

Na Tabela 6 são apresentados os resultados dos ensaios dos corpos de prova de delaminação para as três espécies.

Tabela 6. Valores médios dos ensaios de delaminação para as vigas de MLC

\begin{tabular}{cccc}
\hline Espécie & $\begin{array}{c}\text { Comprimento da } \\
\text { linha de cola }(\mathrm{cm})\end{array}$ & $\begin{array}{c}\text { Delaminação } \\
(\mathrm{cm})\end{array}$ & $\begin{array}{c}\text { Delaminação } \\
(\%)\end{array}$ \\
\hline Tectona grandis & 216 & 1 & 0 \\
Pinus oocarpa & 132 & 0 & 0 \\
Eucalyptus sp. & 216 & 50,5 & 23 \\
\hline
\end{tabular}

Fonte: Autores

Com os resultados apresentados na Tabela 6, é possível avaliar a adesão nas lamelas das vigas de MLC por meio da porcentagem de variação de delaminação da linha de cola. Constatou-se que o tratamento com a espécie Pinus oocarpa apresentou o melhor desempenho entre as três espécies para aplicação estrutural que leve em conta os usos interior e exterior. A espécie Tectona grandis também pode ser indicada para os usos interior e exterior, mesmo apresentando delaminação. O Eucalyptus $s p$. apresentou a maior delaminação, sendo esta combinação espécie-adesivo não recomendada para uso exterior, conforme a The Engineered Wood Association (APA, 2002), e podendo ser indicada apenas para o uso interior. Destaca-se que a espécie Eucalyptus sp. utilizada na fabricação das vigas de MLC apresentou densidade de $872 \mathrm{~kg} . \mathrm{m}-3$ com teor de umidade entre 12 e 14\%. Segundo as prescrições da norma brasileira NBR 7190 (ABNT, 1997), as espécies mais aconselhadas para o emprego em MLC têm valor de referência de densidade até 750 kg.m-3 e são utilizadas com teor de umidade de $12 \%$. Logo, algumas folhosas de baixa densidade podem ser consideradas para a aplicação em MLC, quando facilmente coláveis, tal como Plaster et al. (2008), que apresenta um estudo relacionado ao comportamento de juntas coladas da madeira serrada de Eucalyptus sp., e chegou-se a conclusão que a densidade acima de 800 kg.m-3 influencia a adesão das juntas com relação a porcentagem de falha na madeira, mas os resultados relacionados à resistência ao cisalhamento apresentaram valores satisfatórios nas classes de densidade e suas combinações, confirmando a eficiência da pressão de colagem igual a 1,2 MPa. Segundo Vital, Maciel e Della Lucia (2006), juntas coladas com madeira de Eucalyptus saligna, que apresentou densidade entre 630-660 kg.m-3 com idade de 40 anos, tiveram os valores médios mais elevados de resistência ao cisalhamento e os menores valores de falha na madeira, quando comparadas com Eucalyptus grandis e Pinus elliottii com idades de 50 e 40 anos, respectivamente.

\section{Conclusões}

A partir dos resultados de resistência ao cisalhamento na linha de cola constituída à base de melamina-ureia-formaldeído (Akzo Nobel MUF 1242/2542), concluiu-se que há potencialidade de produção de MLC para ser usada na condição de ambiente seco utilizando a espécie Pinus oocarpa não tratada contra organismos xilófagos; no entanto, a utilização da MLC com esse adesivo em ambiente de alta umidade não é recomendada. As espécies Tectona grandis e Eucalyptus sp. apresentam perda de resistência acentuada na condição seca, sendo necessário mais pesquisas que atestem a viabilidade de uso na construção civil.

Quanto ao ensaio de delaminação, as espécies Tectona grandis e Pinus oocarpa apresentaram 
resultados satisfatórios, indicando a potencialidade do uso estrutural das MLC na construção civil em elementos leves externos, como vigas e pontaletes empregados temporariamente na construção civil, e em elementos pesados internos, como vigas empregadas em coberturas.

O corpo de prova estrutural do tipo push-out, sugerido pela DIN 4074 apud Frangi, Fontana e Mischler (2004), obtido a partir das vigas de MLC apresentou ineficiência para as três espécies de madeira Tectona grandis, Pinus oocarpa e Eucalyptus sp., tratamentos [III] e [IV], porque foi incapaz de representar o modo de ruptura ao cisalhamento observado nos tratamento [I] e [II]. No entanto, na espécie de madeira Pinus oocarpa a resistência do corpo de prova estrutural, tratamento [III], foi representativa comparando com as resistências dos tratamentos [I] e [II].

Conclui-se que as relações geométricas do corpo de prova estrutural de MLC precisam ser reavaliadas na condição seca e úmida por meio de estudos experimentais, a fim de possibilitarem um efeito de cisalhamento puro, tornando-o adequado para todas as espécies de madeira e não apenas representativo do ponto de vista da resistência, como ocorreu para o Pinus oocarpa na condição seca. Sugere-se que os próximos estudos indicando o uso estrutural dos elementos de MLC colados com o adesivo bicomponente de aplicação estrutural à base de melamina-ureia-formaldeído (Akzo Nobel MUF 1242/2542) sejam realizados principalmente em elementos estruturais, vigas de ML, respeitando as relações entre secção transversal e comprimento da viga, bem como ensaios de tração da linha de cola e de emendas dentadas a fim que a indicação desse adesivo seja confirmada.

\section{Agradecimentos}

À empresa Akzo Nobel Ltda., pela disponibilidade em ceder a amostra de adesivo bicomponente à base de melamina-ureia-formaldeído para realização desta pesquisa.

\section{Referências}

AMERICAN INSTITUTE OF TIMBER CONSTRUCTION. AITC T110: test methods for structural glued laminated timber. Colorado: AITC, 2007.

AITC T115: machining test for end joints. Colorado: AITC, 2004.

ASSOCIAÇÃO BRASILEIRA DE NORMAS TÉCNICAS - ABNT. NBR 7190: projeto de estruturas de madeira. Rio de Janeiro: ABNT, 1997.

ASSOCIAÇÃO BRASILEIRA DE PRODUTORES DE FLORESTAS PLANTADAS - ABRAF. Anuário estatístico da ABRAF 2012 ano base 2011. Brasília: ABRAF, 2012.

CANADIAN STANDARDS ASSOCIATION. CAN/CSA 0177: qualification code for manufactures of structural glued-laminated timber. Ontario: CSA, 2006.

DIAS, A. A.; LAHR, F. A. R. Maderas tropicales brasileñas alternativas. Maderas: Ciencia y Tecnología, v. 1, n. 2, p. 7-14, 1999.

FAHERTY, K. F.; WILLIAMSON, G. Wood engineering and construction handbook. 3thed. New York: McGraw-Hill Publishing Company, 1997.

FRANGI, A.; FONTANA, M.; MISCHLER, A. Shear behaviour of bond lines in glued laminated timber beams at high temperatures. Wood Science and Technology, v. 38, n. 2, p. 119-126, 2004.

LEVENE, H. Robust Test for the equality of variance. In. OLKIN, I. (Ed.). Contribution to Probability and Statistcs. Palo Alto: Stanford University Press, p. 278-292, 1960.

MACEDO, N. A.; DIAS, A. A.; BARATA, T. Q. F. Madeiras tropicais da Amazônia. In: ENCONTRO BRASILEIRO DE MADEIRA E ESTRUTURAS EM MADEIRA - EBRAMEM, 7., 2000, São Carlos. Anais... São Carlos, 2000. CD-ROM. 
MOODY, R. C.; HERNANDEZ, R.; LIU, J. Y. Glued structural members. In: WOOD HANDBOOK: Wood as an engineering material. Madison: Forest Products Laboratory, 1999. Gen. Tech. Rep. FPL-GTR-113, chapter 11.

PLASTER, O. B.; OLIVEIRA, J. T. S.; ABRAH ̃̃O, C. P.; BRAZ, R. L. Comportamento de juntas coladas da madeira serrada de Eucalyptus sp. CERNE, v. 14, n. 3, p. 251-258, 2008.

SANTOS, C. M. T.; DEL MENEZZI, C. H. $\mathrm{S}$. Efeito da gramatura sobre a resistência ao cisalhamento da linha de cola de duas madeiras tropicais: seru (Allantomalineata) e marupá (Simaroubaamara). Floresta, v. 40, n. 2, p. 345354, 2010.

SHAPIRO, S. S.; WILK, M. B. An analysis of variance test for normality (complete sample). Biometrika, v. 52, p. 591-611, 1965.

SZÜCS, C. A. Painéis - MLC. 2006. Disponível em: $\quad<$ http://www.remade.com.br/br/madeira_ paineis.php? num $=14 \&$ title $=$ MLC $>$. Acesso em: 2 set. 2012.

THE ENGINEERED WOOD ASSOCIATION - APA. Bond classification. Technical topics. Technical Services Division, 2002.

TIENNE, D. L. C.; NASCIMENTO, A. M.; GARCIA, R. A.; SILVA, D. B. Qualidade de adesão de juntas de madeira de pinus coladas em condições simuladas de serviço interna e externa. Floresta e Ambiente, v. 18, n. 1, p. 1629, 2011.

VITAL, B. R.; MACIEL, A. S.; DELLA LUCIA, R. M. Qualidade de juntas coladas com lâminas de madeira oriundas de três regiões do tronco de Eucalyptus grandis, Eucalyptus saligna e Pinus elliottii. Árvore, v. 30, n. 4, p. 637-644, 2006.

ZANGIÁCOMO, A. L. Emprego de espécies tropicais alternativas na produção de elementos estruturais de madeira laminada colada. 2003. Dissertação (Mestrado em Engenharia de Estruturas) - Universidade de São Paulo, São Carlos. Aceito em 11 Setembro, 2014 - Accepted on September 11, 2014. 